\title{
Design of an Op-Amp Free Voltage Reference with PWM Regulation
}

\author{
Pinar Basak Basyurt \\ Dept. of Electronics and \\ Communications Engineering \\ Istanbul Technical University \\ Istanbul, Turkey \\ E-mail: basyurt@itu.edu.tr
}

\author{
Edoardo Bonizzoni, Franco Maloberti \\ Dept. of Electrical, Computer and \\ Biomedical Engineering \\ University of Pavia \\ Pavia, Italy \\ E-mail: edoardo.bonizzoni@unipv.it
}

\author{
Devrim Yilmaz Aksin \\ Analog Devices, Inc. \\ Istanbul, Turkey \\ E-mail: devrimaksin@vlsi.itu.edu.tr
}

\begin{abstract}
This paper presents the design of an op-amp free voltage reference generator which utilizes a pulse width regulated loop. The proposed voltage reference circuit has been designed and simulated in a standard 0.18- $\mu \mathrm{m}$ CMOS technology. Post layout simulation results show that it is capable of operating with supply voltages down to $0.7 \mathrm{~V}$ while generating an output voltage of $202 \mathrm{mV}$. The achieved temperature coefficient is $54 \mathrm{ppm} /{ }^{\circ} \mathrm{C}$ for temperatures ranging from 0 to $100{ }^{\circ} \mathrm{C}$. The simulated power consumption at the room temperature is $400 \mathrm{nW}$.
\end{abstract}

\section{INTRODUCTION}

Voltage references are one of the critical building blocks of many systems on chip (SoCs) and mixed-signal integrated circuits (ICs) such as data converters and voltage regulators since they constitute a stable operating point for the rest of the system in order to generate predictable and repeatable results. Besides being insensitive to temperature, process and supply voltage variations, due to the increasing market demand for battery-operated circuits, ultra-low power consumption and capability to work with very low supply voltage levels have become the most stringent performance parameters for a high performance voltage reference circuit.

Historically, bandgap references (BGRs) have been the most popular solution to generate integrated precision references, [1]. Nowadays, however, the technology scaling and the aforementioned requests of low power and of more and more reduced supply voltage levels make traditional BGR architectures no more suitable for sub- $1 \mathrm{~V}$ operation. Two are the main reasons. The first one is that the BGR output voltage is equal to the extrapolated bandgap voltage of silicon at $0 \mathrm{~K}$, $\approx 1.2 \mathrm{~V}$, that exceeds $1 \mathrm{~V}$. The second limit is posed by the required input common mode voltage level of the amplifier used in the feedback loop that generates the proportional to the absolute temperature (PTAT) current in conventional solutions, [2], [3].

Different approaches have been proposed to overcome these two problems. One of them, [2], uses a resistive subdivision method that allows scaling down the reference voltage in the output branch in order to eliminate the first problem. These circuits are referred as current-mode references since they generate a temperature-independent current, which is then mirrored to an output resistor to create a sub- $1 \mathrm{~V}$ output voltage. However, even if the theoretical usable minimum supply voltage for this architecture is $V_{D D, \text { min }}=V_{E B}+\left|V_{D S \text { sat, } p}\right|$, practically, the required minimum supply voltage is limited by the input common mode range of the op-amp used to generate the PTAT current. To overcome this limitation, several techniques have been published: among them, the use of native (low-threshold) devices, [2], the use of a BiCMOS technology, [4], the use of an amplifier with PMOS input stage driven by a potential divider and with PMOS source-bulk junctions partially forward biased to reduce their threshold voltage, [5], the use of dynamic threshold MOS (DTMOS) devices, [6].

Increasing requirements for reference generators working with very low supply voltage and very low power should be satisfied by new circuit solutions using standard technologies. This work presents the design of an op-amp free voltage reference. The proposed scheme offers a double benefit: it gets rid of the most power hungry block in voltage reference circuits and naturally solves the aforementioned limit imposed by the op-amp input common mode range. The proposed voltage reference circuit uses a reverse current-mode scheme and is regulated with pulse width modulation (PWM). The circuit, designed and simulated at the post layout level with a 2-poly, 6-metal, standard 0.18- $\mu \mathrm{m}$ CMOS technology, achieves an output voltage of $202 \mathrm{mV}$ with a temperature coefficient

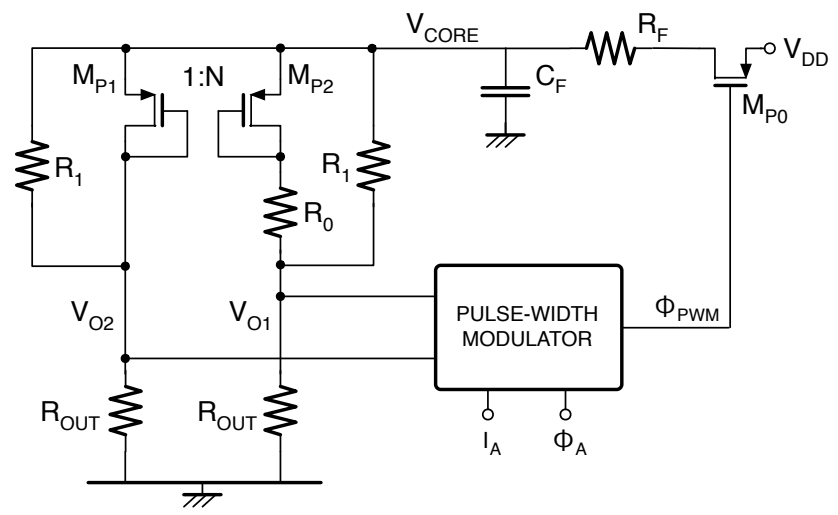

Fig. 1. Schematic diagram of the proposed voltage reference architecture. 


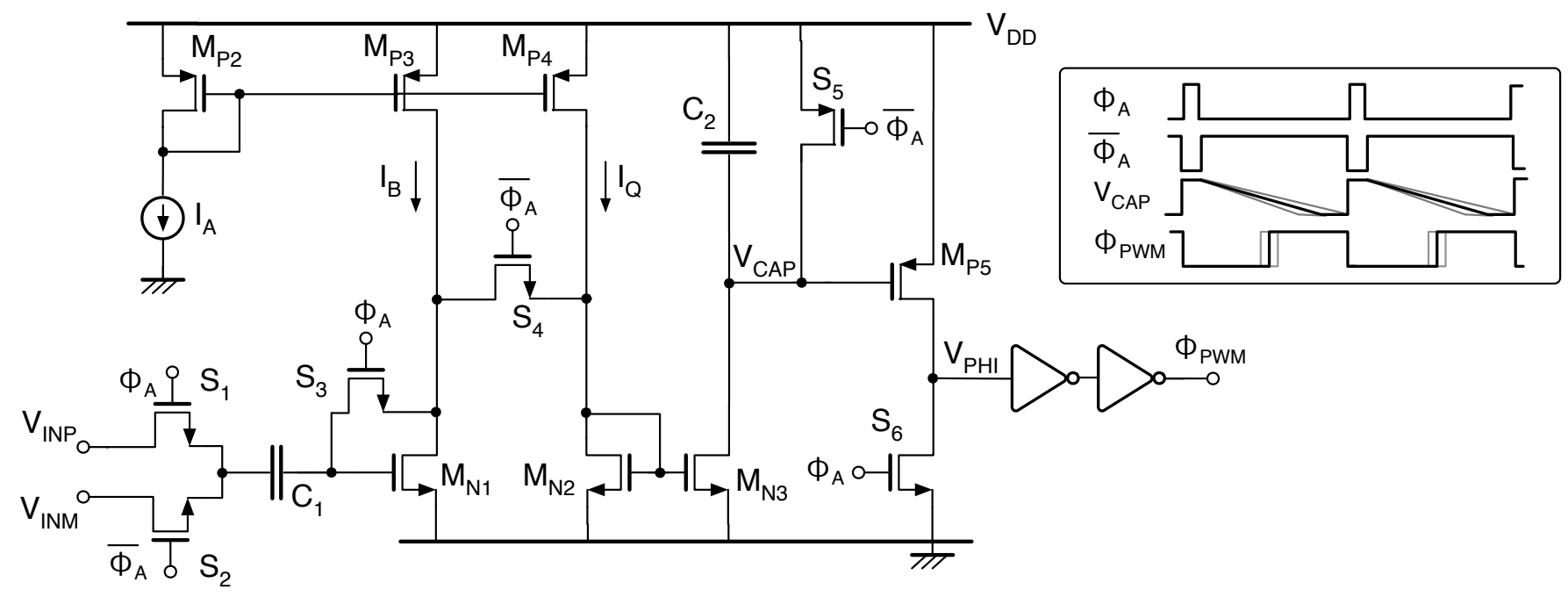

Fig. 2. Schematic diagram of the pulse-width modulator.

(TC) of $54 \mathrm{ppm} /{ }^{\circ} \mathrm{C}$ in the temperature range $0-100{ }^{\circ} \mathrm{C}$ and consumes always less then $500 \mathrm{nW}$ over the entire temperature range with a supply voltage as low as $0.7 \mathrm{~V}$.

\section{Proposed Voltage Reference}

Fig. 1 shows the schematic diagram of the proposed voltage reference circuit. The conventional current mode bandgap, [2], is reversed so that the reference voltage is generated across the output resistors $R_{O U T}$. The direct transformation of PTAT and CTAT currents into voltage with a reversed architecture avoids the use of current mirrors, often source of inaccuracy, [7]. Transistors $M_{P 1}$ and $M_{P 2}$ operate in the sub-threshold region to provide the diode-like voltage-current response, as required to give rise to PTAT and CTAT currents. The PMOS switch $M_{P 0}$, digitally driven by the pulse-width modulator block, generates the voltage $V_{C O R E}$. The action of the simple RC network (composed by $R_{F}$ and $C_{F}$ ) adequately filters out the ripple caused by the PWM on $V_{C O R E}$. The loop equates the two output voltages, $V_{O 1}$ and $V_{O 2}$. The output voltage of the circuit can be expressed as

$$
V_{O 1, O 2}=\frac{R_{O U T}}{R_{1}}\left[V_{G S 1}+\frac{R_{1}}{R_{0}} n V_{T} \ln (N)\right]
$$

With $N=16$ and $R_{1} / R_{0}=2.25$, it is first order temperature independent. $R_{O U T}$ is chosen equal to $1 \mathrm{M} \Omega$ to have an output voltage of $200 \mathrm{mV}$. The operation incorporates the start-up function, which is always required in bandgap circuits.

\section{A. Pulse-Width Modulator}

Fig. 2 shows the schematic diagram of the designed pulse width modulator. There are two phases of operation. In the first phase, $\Phi_{A}$, the input transistor, $M_{N 1}$, is connected in the diode configuration to charge the auto-zero capacitor $C_{1}$ to $V_{I N P}$ minus the $V_{G S}$ of the input transistor itself. In the second phase, $\overline{\Phi_{A}}$, the signal current, $i_{d}$, flows through the switch
$S_{4}$ into $M_{N 2}$ and is mirrored to $M_{N 3}$ in order to charge the capacitor $C_{2}$, initially discharged in the auto-zero phase. In this phase, the voltage $V_{C A P}$ linearly decreases and the $V_{G S}$ of $M_{P 5}$ is compared with the threshold voltage of $M_{P 5}$. The result at the node $V_{P H I}$ is a square wave, which is enforced by a tapered inverter chain to generate the PWM signal at the output. Fig. 2 conceptually shows the operation and provides the phases diagram and the evolution of the voltage $V_{C A P}$. The input differential voltage and the transconductance of the input transistor $M_{N 1}$, which operates in the sub-threshold region, give rise to the charging signal current:

$$
i_{d}=g_{m, M N 1}\left(V_{I N P}-V_{I N M}\right)=\frac{I_{B}}{n V_{T}}\left(V_{I N P}-V_{I N M}\right)
$$

where $I_{B}$ is the bias current of $M_{N 1}, n$ the sub-threshold region slope and $V_{T}$ the thermal voltage.

The quiescent pulse width, $P W_{Q}$, of the generated square wave depends on the quiescent current, $I_{Q}$, the threshold voltage of $M_{P 5}, V_{t h, p, M 5}$, the value of $C_{2}$, the auto-zero duration time, $T_{A}$, and the master clock period, $T\left(T_{A}<T\right)$. With zero signal current, $P W_{Q}$ is equal to

$$
P W_{Q}=T-T_{A}-\frac{C_{2}\left|V_{t h, p, M 5}\right|}{I_{Q}}
$$

In the preferred implementation, the bias current $I_{A}$ is $10 \mathrm{nA}$ and $M_{P 2}$ has $W / L$ equal to 4 . The aspect ratios of transistors $M_{P 3}$ and $M_{P 4}$ are 40 and 8, respectively, to achieve the nominal currents $I_{B}$ and $I_{Q}$ equal to $100 \mathrm{nA}$ and $20 \mathrm{nA}$. The capacitor $C_{1}$ used for storing the input offset during the auto-zero period is $2 \mathrm{pF}$. The clock frequency is $100 \mathrm{kHz}(T=10 \mu \mathrm{s})$ with auto-zero phase, $\Phi_{A}$, equal to $25 \%$ of the clock period $\left(T_{A}=\right.$ $2.5 \mu \mathrm{s})$. From (3), in order to achieve $P W_{Q}=\left(T-T_{A}\right) / 2$, considering $\left|V_{t h, p, M 5}\right|=0.45 \mathrm{~V}$ and $\mathrm{C}_{2}=0.15 \mathrm{pF}$, the quiescent current, $I_{Q}$, turns out to be equal to $18 \mathrm{nA}$. Table I gives the 
TABLE I

Transistor Sizes of the Pulse Width Modulator

\begin{tabular}{c|c}
\hline Transistor & W/L \\
\hline$M_{P 2}$ & $20 \mu \mathrm{m} / 5 \mu \mathrm{m}$ \\
\hline$M_{P 3}$ & $200 \mu \mathrm{m} / 5 \mu \mathrm{m}$ \\
\hline$M_{P 4}$ & $40 \mu \mathrm{m} / 5 \mu \mathrm{m}$ \\
\hline$M_{P 5}$ & $50 \mu \mathrm{m} / 0.18 \mu \mathrm{m}$ \\
\hline$M_{N 1}$ & $100 \mu \mathrm{m} / 1 \mu \mathrm{m}$ \\
\hline$M_{N 2}, M_{N 3}$ & $20 \mu \mathrm{m} / 1 \mu \mathrm{m}$ \\
\hline$S_{1}, S_{2}$ & $0.22 \mu \mathrm{m} / 0.18 \mu \mathrm{m}$ \\
\hline$S_{3}, S_{4}, S_{5}, S_{6}$ & $1 \mu \mathrm{m} / 0.18 \mu \mathrm{m}$ \\
\hline
\end{tabular}

transistors sizes of the designed circuit in the used $0.18-\mu \mathrm{m}$ CMOS technology.

\section{B. Supply Voltage Limit}

The required minimum supply voltage for the proposed circuit depends on the desired output level and a number of practical limits. From Fig. 1, the minimum $V_{D D}$ can be expressed as

$$
V_{D D}=V_{O 1, O 2}+\left|V_{G S, M P 1}\right|+V_{F}
$$

where $V_{F}$ is the voltage drop on the filter resistor when transistor $M_{P 0}$ is on. In our design, an output voltage of $200 \mathrm{mV}, 400 \mathrm{mV}$ across the PMOS diode at $0{ }^{\circ} \mathrm{C}$ (worst case for the target temperature range), and a voltage drop as low as $50 \mathrm{mV}$ across $R_{F}$ result in a minimum supply voltage equal to $0.65 \mathrm{~V}$.

On the other hand, proper operation for switches $S_{1}$ and $S_{2}$ of Fig. 2 has to be ensured in order to avoid significant circuit performance degradation. This leads to the following additional constraint:

$$
V_{D D}=V_{O 1, O 2}+V_{t h, n}+V_{D S s a t}
$$

In the used technology, the threshold of the N-channel transistors is $450 \mathrm{mV}$ at $0{ }^{\circ} \mathrm{C}$ (that is the worst case for the target temperature range). Hence, with an overdrive voltage of $50 \mathrm{mV}$, the minimum required supply voltage becomes $0.7 \mathrm{~V}$. However, this constraint can be eliminated if the driving signals of the two switches are properly boosted, for instance by means of simple voltage doubler circuits, [8].

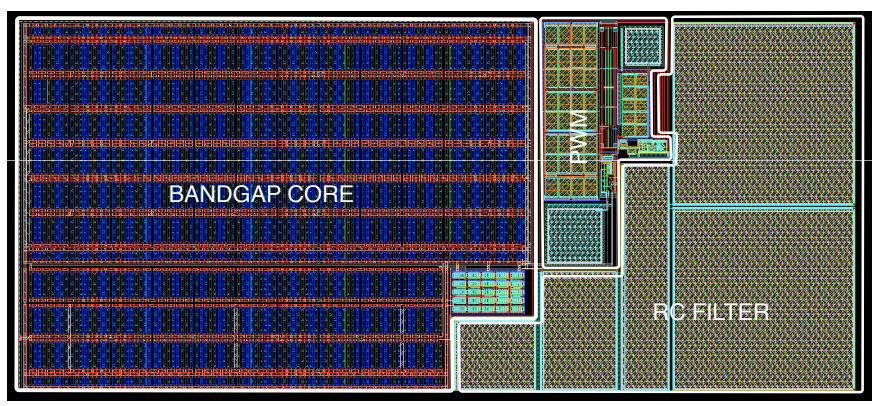

Fig. 3. Layout of the proposed voltage reference circuit.
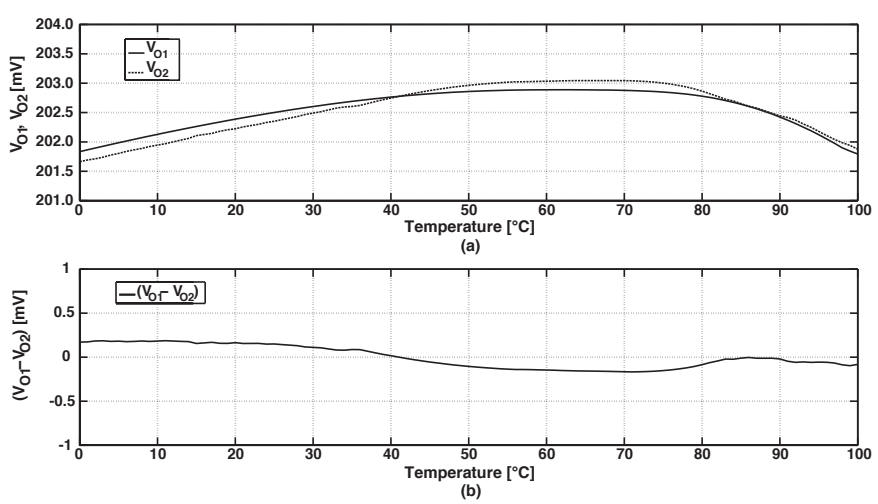

Fig. 4. Post layout simulation of (a) the two output voltages and (b) of their difference as a function of the temperature.

\section{Simulation Results}

This voltage reference has been designed and simulated at the post layout level in a standard $0.18-\mu \mathrm{m}$ CMOS technology with 6 metal and 2 poly layers. Fig. 3 shows the layout of the circuit with main blocks highlighted. The circuit occupies an active area of $370 \mu \mathrm{m} \times 170 \mu \mathrm{m}$ that is mainly dominated by the resistors used in the bandgap core and by the capacitor of the RC filter. The nominal supply voltage and the master clock frequency are $0.7 \mathrm{~V}$ and $100 \mathrm{kHz}$, respectively. The auto-zero phase, $\Phi_{A}$, is set to $25 \%$ of the master clock period.

Fig. 4 shows the two output voltages and their difference as a function of the temperature. The circuit generates two output voltages of about $200 \mathrm{mV}$ in the nominal process corner. In the temperature range from $0{ }^{\circ} \mathrm{C}$ to $100{ }^{\circ} \mathrm{C}$, the difference between the two outputs is less then $\pm 0.5 \mathrm{mV}$. For this temperature range, the achieved TCs of the outputs, $V_{O 1}$ and $V_{O 2}$, are $54 \mathrm{ppm} /{ }^{\circ} \mathrm{C}$ and $62.5 \mathrm{ppm} /{ }^{\circ} \mathrm{C}$, respectively.

Fig. 5 plots the distribution of the output voltage levels for the two outputs obtained through 100 process and mismatch Monte Carlo simulation runs without any device trimming or current calibration for the PWM circuit. The mean values of the two output voltages are $\approx 202 \mathrm{mV}$ with standard deviations of $1.665 \mathrm{mV}$ and $1.717 \mathrm{mV}$, respectively.
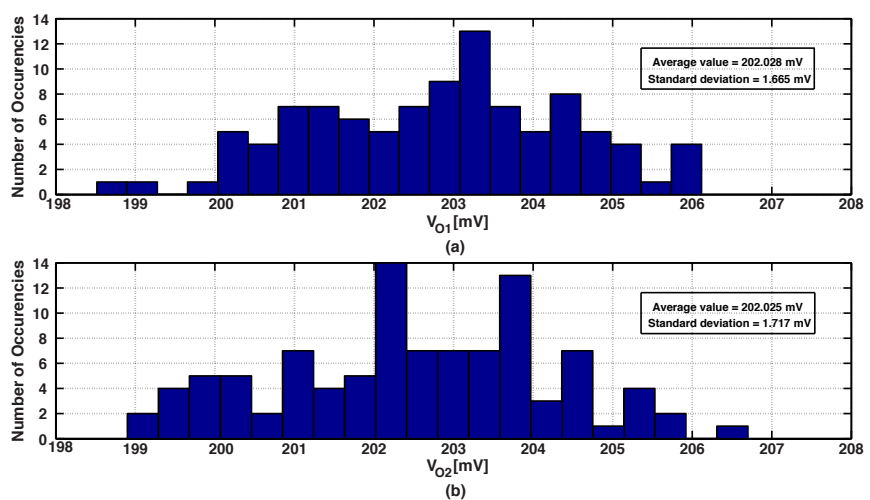

Fig. 5. Untrimmed output voltages level distribution achieved with 100 Monte Carlo simulation runs. (a) $V_{O 1}$; (b) $V_{O 2}$. 


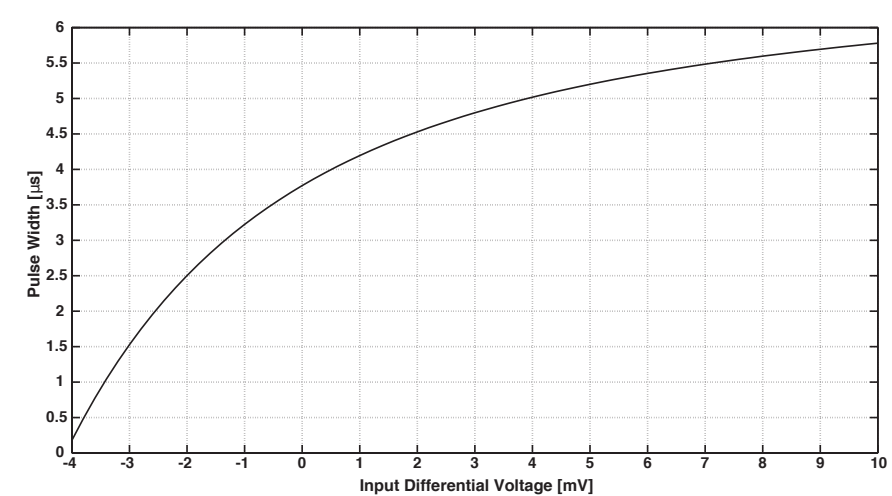

Fig. 6. Post layout simulation of the PWM signal pulse width as a function of input differential voltage.

Fig. 6 gives the pulse width of the generated PWM signal as a function of input differential voltage (input-output characteristic of the PWM circuit). Note that, with a negative input signal, the current charging the capacitor $C_{2}$ diminishes, thus increasing the time required to the voltage $V_{C A P}$ for crossing the value $V_{D D}-\left|V_{t h, p, M P 5}\right|$. The pulse width of the generated signal on node $V_{P H I}$ (and, consequently, $\Phi_{P W M}$ ) decreases. A positive input signal, on the contrary, speeds up charging $C_{2}$ and the pulse width of the output signal becomes larger.

Fig. 7 shows the quiescent pulse width of the PWM signal, $P W_{Q}$, as a function of the temperature in the range from 0 to $100{ }^{\circ} \mathrm{C}$. The positive slope of the curve is caused by two effects, both ascribed to the transistors threshold temperature dependence. The first and dominant one is the threshold voltage variation of $M_{P 5}$, that decreases the charge needed on capacitor $C_{2}$, hence, increasing the output pulse width. The second effect is the variation of the threshold of the transistors composing the current mirrors $\left(M_{P 3}-M_{P 4}\right.$ and $M_{N 2}-$ $M_{N 3}$ ) which provide the quiescent bias current. The latter increases with temperature.

Fig. 8 shows the supply current as a function of the temperature when the nominal supply voltage is $0.7 \mathrm{~V}$. The supply current is increasing with temperature because of the CTAT behavior of the $V_{G S}$ of PMOS transistors, $M_{P 1}$ and $M_{P 2}$. The average value of the supply current is $590.5 \mathrm{nA}$. At room

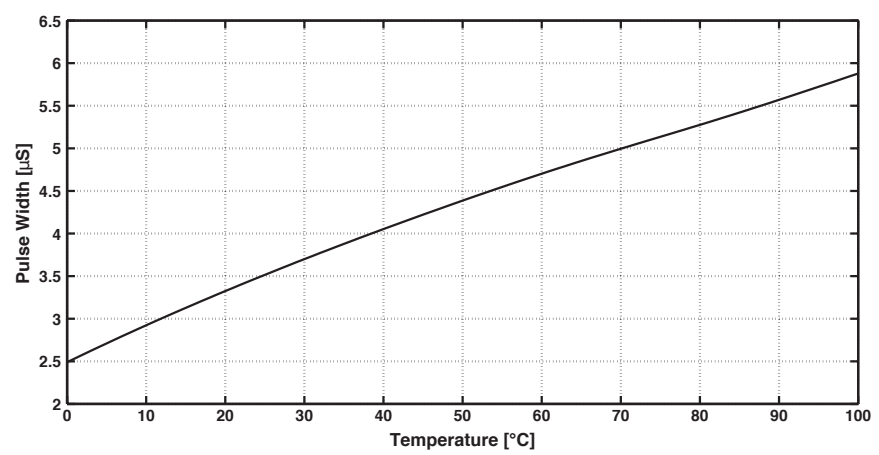

Fig. 7. Post layout simulated pulse width variation as a function of temperature.

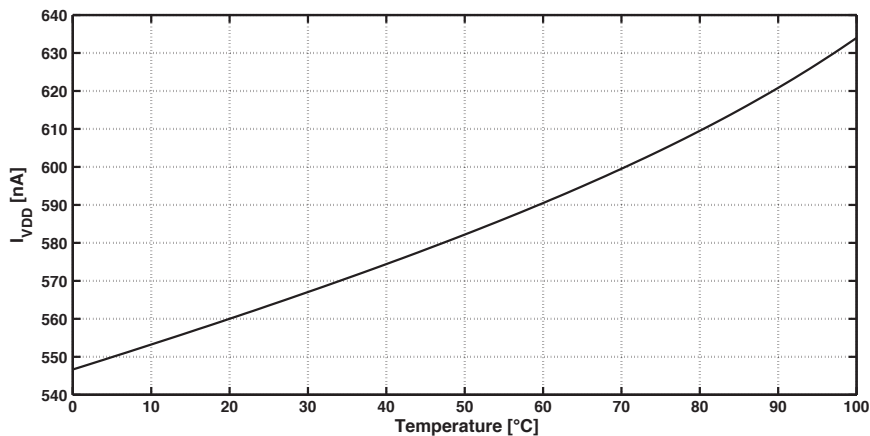

Fig. 8. Supply current variation as a function of temperature simulated at the post layout level.

temperature, the supply current is about $565 \mathrm{nA}$, thus bringing the power consumption to $395.5 \mathrm{nW}$. Even for the worst case (at $100{ }^{\circ} \mathrm{C}$ ), the power consumption is less then $0.5 \mu \mathrm{W}$.

\section{Conclusion}

This paper presented a novel low power voltage reference circuit implemented without any operational amplifier. The circuit is regulated with pulse width modulation. The scheme, designed and simulated at the post layout level with a standard $0.18-\mu \mathrm{m}$ CMOS technology without any special process step, is capable of operating with very low supply voltage and achieves a temperature coefficient of $54 \mathrm{ppm} /{ }^{\circ} \mathrm{C}$ in the temperature range $0-100{ }^{\circ} \mathrm{C}$. The simulated power consumption is always less than $500 \mathrm{nW}$.

\section{ACKNOWLEDGMENT}

This work is partially supported by The Scientific and Technological Research Council of Turkey under the International Research Fellowship Programme-2214.

\section{REFERENCES}

[1] G. A. Rincon-Mora, "Voltage References: from Diodes to Precision Higher-Order Bandgap Circuits", New York: Wiley-IEEE, 2001.

[2] H. Banba, H. Shiga, A. Umezawa, T. Miyaba, T. Tanzawa, S. Atsumi, and K. Sakui, "A CMOS Bandgap Reference Circuit with sub-1V Operation", IEEE Journal of Solid-State Circuits, vol. 34, no. 5, pp. 670-674 May 1999.

[3] Y. Jiang and E. K. F Lee, "Design of Low-voltage Bandgap Reference Using Transimpedance Amplifier", Circuits and Systems II: Analog and Digital Signal Processing, IEEE Transactions on, vol. 47, no. 6, pp. $552-$ 555, 2000.

[4] P. Malcovati, F. Maloberti, C. Fiocchi, and M. Pruzzi“"CurvatureCompensated BiCMOS Bandgap with 1-V Supply Voltage", IEEE Journal of Solid-State Circuits, vol. 36, no. 7, pp. 1076 -1081, July 2001.

[5] K. N. Leung and P. K. T Mok, "A Sub-1-V 15-ppm/ ${ }^{\circ} \mathrm{C}$ CMOS Bandgap Voltage Reference without Requiring Low Threshold Voltage Device", IEEE Journal of Solid-State Circuits, vol. 37, no. 4, pp. 526-530, Apr. 2002.

[6] A. J. Annema,, "Low-power Bandgap References Featuring DTMOSTs", IEEE Journal of Solid-State Circuits, vol. 34, no. 7, pp. 949 -955, Jul. 1999.

[7] V. Ivanov, R. Brederlow, and J. Gerber, "An Ultra Low Power Bandgap Operational at Supply from 0.75 V", IEEE Journal of Solid-State Circuits, vol. 47, no. 7, pp. 1515-1523, July 2012.

[8] P. Favrat, P. Deval, M.J. Declercq, "A High-Efficiency CMOS Voltage Doubler", IEEE Journal of Solid-State Circuits, vol. 33, no. 3, pp. 410416, 1998. 